\title{
Systematic Review of Barriers and Facilitators to Venous Thromboembolism Clinical Practice Guideline Compliance by Healthcare Professionals in Acute Care
}

\author{
Sherryl Gaston ${ }^{1, *}$, Sarahlouise White ${ }^{2}$ \\ 1Nursing and Rural Health, University of South Australia: Centre for Regional Engagement \& The Joanna Briggs Institute: Faculty \\ of Health Sciences, The University of Adelaide, South Australia \\ 2The Joanna Briggs Institute, School of Translational Health Science, Faculty of Health Sciences, The University of Adelaide, \\ South Australia \\ *Corresponding Author: sherryl.gaston@unisa.edu.au
}

Copyright (C) 2013 Horizon Research Publishing All rights reserved.

\begin{abstract}
Aims: To examine compliance with Venous Thromboembolism clinical practice guidelines by health professionals and to identify barriers and facilitators in the acute healthcare setting. Background: Clinical practice guidelines for venous thromboembolism prevention are widely available, however not all patients with identifiable risk factors are receiving appropriate assessment or prophylaxis in the acute care setting. Design: Joanna Briggs Institute comprehensive systematic review methodology was utilised. Data Sources: A comprehensive, three-stage search strategy was conducted across six major databases. Review Methods: Retrieved papers were critically appraised by two independent reviewers prior to inclusion using standardised critical appraisal instruments. Results: Eighteen quantitative and two qualitative studies assessed compliance with venous thromboembolism clinical practice guidelines, and reported on facilitators and barriers to that compliance. Quantitative data was pooled using narrative summary due to heterogeneity of reporting. Qualitative data was aggregated using a process of meta-aggregation to generate synthesised findings based on similarity of meaning. Overall, nine barriers and nine facilitators were identified Conclusion: This systematic review has drawn together quantitative and qualitative data that highlights barriers to compliance and recommends that if an intervention is developed with these in mind they can improve compliance with clinical practice guidelines.
\end{abstract}

Keywords Barriers, Compliance, Continuous quality improvement,Facilitator, Healthcare Professional, Systematic Review, Venous Thromboembolism

\section{Introduction}

Venous Thromboembolism (VTE) is the term used to describe the two conditions Deep Vein Thrombosis (DVT) and Pulmonary Embolism (PE)[1]. DVT usually develops as the result of a blood clot forming in the deep veins of the leg but can sometimes form in the pelvis[1]. A PE occurs when there is an obstruction in the pulmonary artery or its branches in the lungs, most often is the result of a clot in the leg either breaking away or part of it breaking off and moving from the vein to the lungs[1].

There are two main categories of risk factors for VTE. The first category is associated with the clinical setting such as surgery and type of surgery. Examples include - major surgery with medical risk factors, immobilisation and immobilising treatments, medical patients with additional risk factors, some fractures, stroke, some chemotherapy treatment, acute spinal injury or multiple trauma[2]. The second category refers to patient factors such as age, previous history of DVT/PE, past history of major surgery, recent pregnancy, malignancy, obesity, oral contraceptive or hormone replacement, inflammatory bowel disease, as well as any thrombophilia conditions[2].

VTE is of global concern: in 2008, 14,716 cases of VTE were reported across Australia[3]. This represents an increase in the rate of hospital separations for VTE in Australia of 2 per 100,000 since 2004/05[3]. It is estimated that annually in Australia about 30,000 hospitalisations are due to VTE and that about 5,000 people in Australia die as a result[4]. PE is reported as being responsible for $10 \%$ of all hospital deaths in Australia[5].Estimates of 25,000 [6] (UK) and 300,000[7](USA) VTE-related deaths per year have been reported.

Morbidity for survivors of VTE can be quite debilitating and some of the complications are not apparent until weeks or months after the initial blood clot[8]. These complications are caused by compromised blood flow from the lower leg veins back to the heart[3].

The incidence of VTE in hospitalised patients is higher than that of people living in the community who have similar demographics, and can be as much as 100 times higher[1, 2]. In 2008 the National Institute of Clinical Studies (NICS) reported that many of the deaths attributed to VTE may be 
prevented if patients are properly assessed for VTE risk, and appropriate prophylaxis is initiated[2].Proper risk assessment and management based on that assessment including provision of any appropriate VTE prophylaxis is recommended[1,2,9].

An optimal way to prevent VTE in the acute setting is to complete a VTE risk assessment on patients when they are admitted and when their circumstances change, and then provide prophylaxis appropriate to the level of risk identified[2, 9, 10]. A risk assessment tool is used to identify indicators of potential risks using standard criteria[11].Risk assessment tools should be evidence based and usually incorporate a scoring system weighted for particular risk factors that when combined give an overall risk score[12].

Evidence-based clinical practice guidelines are developed to bridge the gap between clinical practice and research, and healthcare professionals use these tools to improve patient care with recognised standards to guide decision making processes[13, 14]. These tools are generally developed following a review of available evidence in association with clinical experts[13]. By producing national guidelines and encouraging healthcare facilities and professionals to adoptthem, there is an attempt at standardisation of patient care, implementation of evidence based healthcare decisions, resulting ultimately in improvements in the quality of delivered care[13].

Even though venous thromboembolism clinical practice guidelines are widely available and have been in existence for several years, their recommendations do not always translate into clinical practice[14, 15], leading to variation in the quality of care provided within a healthcare facility as well as between different organisations[16].Reasons for clinical guidelines not being adhered to(barriers) by healthcare professionals is likely to be multifaceted and knowledge of such barriers can lead to development of targeted strategies to address them to improve compliance and subsequent patient outcomes. Improvements in compliance can be monitored and assessed with clinical audits[16].

\section{Methods}

\subsection{Aims}

The objective of this comprehensive systematic review was to identify, appraise and synthesise the best available evidence of compliance with clinical practice guidelines for VTE risk assessment and prophylaxis and to identify facilitators and barriers to this compliance.

\subsection{Design}

Due to the broad nature of the topic, this systematic review considered both quantitative and qualitative research evidence.

The quantitative component of the review considered both experimental and epidemiological study designs including randomised controlled trials, non-randomised controlled trials, quasi-experimental, pre-test post-test studies, before and after studies, prospective and retrospective cohort studies, case control studies and analytical cross sectional studies.

The qualitative component of the review considered studies that focused on qualitative data including, designs such as phenomenology, grounded theory, ethnography, action research and feminist research.

This review was conducted in accordance with Joanna Briggs Institute and PRISMA guidance.

\subsection{Search Methods}

A three-stage search strategy was utilised. Firstly, a search of MEDLINE and CINAHL was conducted to identify appropriate search words/terms to be used in stage 2 . The second stage was an extensive search using all identified keywords and index terms across all of the included databases. Thirdly, the reference lists of all the literature retrieved from stage 2 were hand-searched for additional studies.

\subsection{Inclusion Criteria}

This review only considered studies published in the English language due to a lack of translational resources. Considered studies were published between May $1^{\text {st }} 2003$ and $30^{\text {th }}$ November 2011 to avoid potential duplication of an existing systematic review on an overlapping topic [17].

Quantitative and qualitative studies were considered for inclusion against defined PICO criteria as detailed below:

Participants: any acute healthcare professional of any specialty or designation

Focus of Interest: compliance or adherence with any published evidence-based VTE clinical guidelines, as well as barriers and/or facilitators to that compliance.

Context: acute care setting

Outcomes: 1) any measure of compliance, 2) anything that could be considered as being a barrier. For the purposes of this review a barrier was defined as anything that inhibits or hinders a healthcare professional conducting a VTE risk assessment and/or providing appropriate prophylaxis 3) anything that could be considered as being a facilitator. For the purposes of this review a facilitator was defined as anything that allowed or helped a healthcare professional to conduct a VTE risk assessment and/or providing appropriate prophylaxis.

\subsection{Initial Search Terms}

Venous Thromboembolism (VTE, DVT, PE); Risk Assessment; Guidelines; Compliance; Adherence; Prophylaxis; Nurse/Doctor; Healthcare worker/professional.

Healthcare professional covered a variety of job descriptions and included: physicians (different levels and specialities also encompassing country-specific terminology such as; doctor, medical practitioner, hospitalists, medical officer, junior doctor, consultant, resident, house staff, medical staff, attending physicians), physician assistants, pharmacists, nurses, nurse practitioners, administrators, health care assistants. 

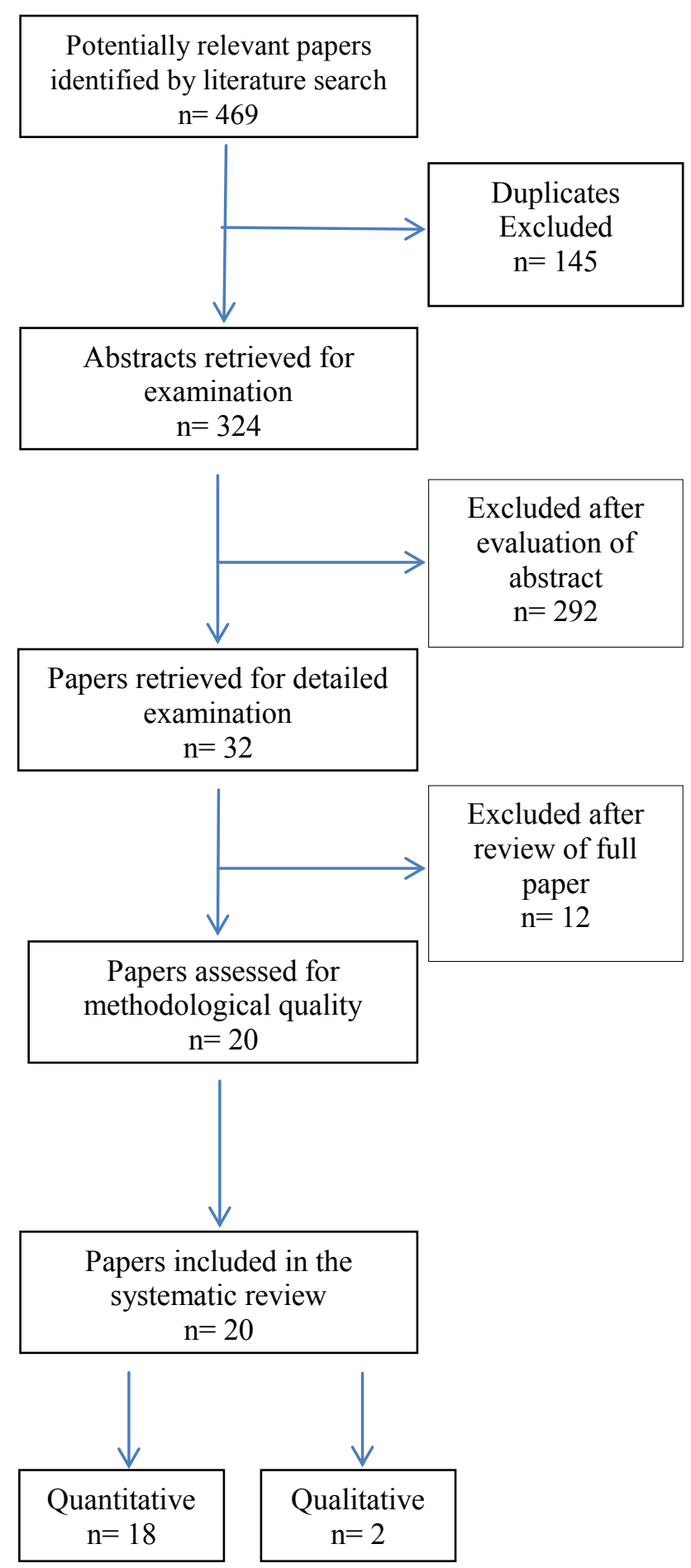

Figure 1. Flowchart detailing study identification

\subsection{Search Outcome}

Initially 469 studies were identified as being potentially relevant to the review question. There were 145 duplicates which were removed, leaving 324 studies. Of the 324 articles, 292 were excluded on the basis of not meeting the inclusion criteria after reading the abstracts. Full text articles of the remaining 32 titles were retrieved and examined thoroughly. After this process 20 articles were found to meet the inclusion criteria and were then critically appraised for methodological quality. Twelve studies were excluded after reading the full text. Eighteen of the included studies utilized quantitative study designs and two studies utilized qualitative research methods, all studies were appraised using the Joanna Briggs Institute critical appraisal tools. The flowchart in Figure 1 details the study identification process. 


\subsection{Quality Appraisal}

Twenty studies were critically appraised by two independent reviewers against standardised checklist criteria. Quantitative papers were assessed using standardised critical appraisal instruments from the Joanna Briggs Institute Meta-Analysis of Statistics Assessment and Review Instrument (JBI-MAStARI). Qualitative papers were assessed using standardised critical appraisal instruments from the Joanna Briggs Institute Qualitative Assessment and Review Instrument (JBI-QARI). There were no disagreements between the reviewers and a third reviewer was not required. Copies of the JBI tools are available from the author on request, or from the Joanna Briggs Institute website.

No studies were excluded following critical appraisal, however methodological quality varied. There was a discussion on the overall usefulness of including studies that were of poorer methodological quality and the decision was made to include all of the studies, regardless of its quality as excluding a study on the basis of methodological quality may lead to lack of identification of important barriers and facilitators.

Sources of potential bias resulting from methodological issues are discussed later.

\subsection{Data Abstraction}

Quantitative data was extracted from papers included in the review using the standardised data appraisal tool from JBI-MAStARI. Qualitative data was extracted from papers included in the review using the standardised data appraisal tool from JBI-QARI. Copies of the JBI tools are available from the author on request, or from the Joanna Briggs Institute website.

Data extracted included specific details about the interventions, populations, study methods and outcomes of significance to the review question and specific objectives.

\subsection{Synthesis}

Quantitative research findings were heterogeneous and it was inappropriate to synthesise them in meta-analysis, therefore findings are presented in narrative summary with the aid of tables.

Qualitative research findings were pooled using JBI-QARI. This involved the aggregation/synthesis of findings to generate a set of statements that represented that aggregation, through assembling the findings they were assigned a level of credibility, and were categorised on the basis of similarity in meaning. These categories were then subjected to a meta-aggregation in order to produce a single comprehensive set of synthesised findings to be used as a basis for evidence-based practice. Copies of the JBI tools are available from the author on request, or from the Joanna Briggs Institute website.

\section{Results}

The twenty studies included in this systematic review used a variety of study designs:sixteen quasi-experimental (pre-test post-test) studies,[1, 18-32] one cohort study[33], one case series[34], one ethnographic study[35] and one study used grounded theory[36] methods.

The studies examined several category of healthcare professional: Medical Practitioners (including the titles of: physicians, medical officers, junior doctor, consultant, residents, house staff, medical staff, attending physicians and doctors), nurses, nurse practitioners, pharmacists, physician assistant, healthcare assistant and hospital administrators.

Seventeen of the studies examined compliance to VTE clinical guidelines for physicians, comprising all titles that are used for this position in the different countries including physician assistant [18-23, 26-36]. Of these seventeen studies, eleven exclusively involved physicians and six included physicians and other healthcare professionals. Nine studies examined compliance with VTE clinical guidelines for nurses and of these three exclusively considered nurses[1, $18,19,22,24,25,31,34,36]$.Five studies examined compliance with VTE clinical guidelines for pharmacists or pharmacy staff. None of these however, were exclusive to this healthcare professional group $[18,22,31,34,36] . S i x$ studies addressed compliance with VTE clinical guidelines for multiple healthcare professional groups $[18,19,22,31,34$, 36].

The search identified the use of three best practice evidence-based guidelines. The three guidelines have similar recommendations and all agree that it is important to provide appropriate prophylaxis for VTE as this will reduce the morbidity and mortality from this condition, ultimately reducing the cost of diagnosis and treatment in the long term[37-39].The American ACCP guideline was utilised by ten different studies[20, 22-24, 26, 27, 29-32], the Australia and New Zealand guideline was utilised by three difference studies[18, 25, 35] and the United Kingdom expert working group guidelines by one study[19]. The other studies either did not specify the guidelines used[34, 36], or simply reported the guidelines used as being hospital guidelines[28], evidence based consensus guidelines[21], venous thromboembolism prevention guidelines[1],or existing consensus guidelines[33].

Ten of the eighteen quantitative studies were conducted in the United States of America, [21-24, 27, 29-31, 33, 34] four in Australia[1, 18, 25, 28] and one each in Saudi Arabia,[26] United Kingdom,[19] Iran[20] and Ireland[32]. The two qualitative studies were conducted in Canada[36] and Australia[35].Twelve of the eighteen included studies were conducted in a metropolitan location with no studies conducted in a rural or remote area.

From the quantitative studies nine barriers and nine facilitators were identified. From the qualitative studies, three barriers and three facilitators were identified. There was overlap between the types of evidence. 


\subsection{Barriers}

Quantitative studies

Healthcare professional lack of attention[18-22] was identified as a barrier to compliance. Studies reported a lack of staff attention to VTE risk assessment and prophylaxis initiation. VTE prevention and management was reported as being not being very high on their list of actions to take in relation to patient care. Healthcare professionals often stated they were too busy to add this to their practice or they simply forgot to complete the requirements for VTE care.

Lack of awareness [1, 18, 19, 23-25, 27, 32] was identified as a barrier. This category covered staff being unaware of what action they should take if a patient had a contraindication to treatment and therefore tended to ignore VTE management or prevention strategies. Medical practitioners were also unaware of when to prescribe VTE prophylaxis for a patient. Some healthcare professionals believed that there were no problems in their practice area even though the assessment of patients who may be at risk was not uniformly completed.

Patient factors [19, 24, 26, 27, 33] were reported as barriers to VTE assessment and prophylaxis and related to healthcare professional concerns about complications with bleeding. There was also a reluctance to prescribe chemical prophylaxis due to the possibility of an adverse reaction or an interaction with other medication the patient may be taking. Healthcare professionals stated that the patient was too ill at times with the focus on addressing their immediate needs and this led to VTE prevention not being seen as a priority.

Knowledge, experiences with and/or access to computers and databases [22, 28, 33] was identified as being a barrier and refers to the observation that some computer applications cannot be used in all hospitals due to software incompatibilities as well as a lack of capability of some systems. Two studies stated that different computer systems used different languages therefore a program developed for one didnot work on another [28, 33]. Computer applications were seen to be quite limiting and not capable of carrying out the task required [22].

Disputing evidence/guidelines [18, 24, 29]was reported as being a barrier where healthcare practitioners felt that the evidence in the guidelines is incorrect. This was reported as the reason for inconsistent use of VTE risk assessment between practitioners as well as between wards, specialties and hospitals.

Lack of documentation [30, 33]was recognised as a barrier due to incomplete coding of patient conditions, leading to a risk assessment document not being completed. It was also noted that some hospitals use varying terms to categorise their patients being at high risk instead of using standard terms based on evidence-based clinical practice guidelines.

Staff factors $[1,19,24]$ were reported as being a barrier and described situations where staff did not know who was responsible for VTE initiatives. It was reported that nurses did not complete the risk assessment because they felt it was the doctor's responsibility, and also that doctors disregarded the recommendations provided by the nurse case manager.

Another system level barrier was lack of system support $[18,34]$ which was seen to occur where there were no developed guidelines for VTE within the health service and there were no risk assessment tools to use. There was also confusion with the risk assessment model developed.

The ninth barrier was reported as being financial constraints[31]where the costs associated with providing staff education was limiting, especially since a high amount of studies support continued and regular staff education.

Qualitative studies

Eleven different findings were extracted from the two included qualitative studies on barriers to compliance with Clinical Practice Guidelines. Analysis of these findings generated three categories of barriers and a subsequent single synthesised finding.

The first category 'Costs and Priority' [36] was comprised off our findings and acknowledges that clinicians were more focused on treating the immediate condition of a patient rather than on preventive care. The findings revealed that if VTE prophylaxis was not initiated early in a patient's admission then it could be overlooked for the remainder of a patients stay. It was recognised that clinicians working within a speciality did not consider treatments needed outside of this focus, such as VTE prophylaxis. Anti-embolic stockings and pneumatic compression were reported as having cost restrictions, there were difficulties with fitting them, patients felt they were inconvenient and became non-compliant.

The second category which contained five findings was lack of role identification [35, 36].There were situations were multiple practitioners were responsible for a patients care, however no-one undertook VTE assessment/prevention as no one person or clinician group felt responsible for completing a VTE risk assessment and initiating prophylaxis. There was a lack of consistency and clarity over responsibility for VTE assessment and prophylaxis. One study stated that $36 \%$ of patients identified as high risk for VTE being overlooked for prophylaxis even though healthcare professionals believed implementation of VTE was appropriate in their facility[35].It was also identified that there was a lack of knowledge in relation to mobilization requirements for patients.

The third category Practice culture[35]was comprised of two findings and included the observation that practice was tailored to what senior members of the team wanted, and junior staff would go along with this rather than using evidence-based clinical practice guidelines provided. Past experience by the clinicians impacted on the preferences they developed for prescribing VTE prophylaxis rather than using clinical practice guidelines.

The three barrier categories were drawn together to generate one synthesized finding titled 'Barriers to compliance with venous thromboembolism clinical practice guidelines'. The accompanying description of this synthesis is that any action or inaction that reduces a clinician's adherence with evidence-based clinical practice guidelines 
for venous thromboembolism prevention and management can be considered as a barrier.

\subsection{Facilitators}

Quantitative studies

A range of strategies were utilised in the quantitative studies to facilitate an improvement in compliance, and were grouped under nine main categories. These were;

Education [1, 18, 23, 25-28, 31]was identified as a facilitator by several studies. Education delivery ranged from sessions conducted face to face within the hospital as a regular occurrence or once off for clinicians, to education outreach visits that supported the clinicians in their work environment.

The second category was computer applications $[21,22$, 27-29, 33, 34]with facilitators such as electronic alerts and prompts to remind clinicians, as well as integration of a VTE risk assessment into the electronic patient record and admission system.

The third category was audit and feedback $[1,18,23,28$, 32] where clinical audits were conducted at regular intervals and the results reported back to clinicians.

The fourth category was reminders [18, 20, 26, 31] with facilitators in this category ranging from daily electronic reminders, stickers placed on case notes to newsletters sent directly to all of the physicians practicing at the facility.

The fifth category was multiple interventions $[25,32,34]$ interventions in this category employed two or more strategies to improve compliance. This included combinations of education, audit and feedback, reminders and allocating a dedicated VTE clinician.

The sixth category was having a dedicated personorclinician group $[1,24,30]$ being responsible for the prevention and management of VTE. This type of intervention was successful in improving both compliance with clinical practice guidelines as well as patient outcomes.

The seventh category of facilitators was system support $[18$, $21,34]$ where studies assessed the effectiveness of providing system supports to conducting VTE risk assessment such as pre-printed orders as well as the development and standardisation of care policy, procedures and tools.

The eighth category was labeled management incorporation [26, 31] where the effectiveness of incorporating VTE education and reporting into regular quality meetings and hospital rounds was found to facilitate an improvement in compliance with clinical practice guidelines.

The ninth category was pharmacy involvement [31]improvement in compliance was reported when pharmacists were involved in the hospital rounds, as well as the pharmacy department providing prompts for chemical prophylaxis in combination with complication and implications.

Qualitative studies.

The qualitative studies identified seven findings, subsequently grouped together by the JBI-QARI tool, under three categories with their facilitators to improvement in compliance with VTE clinical practice guidelines. The facilitators highlighted by one of the qualitative studies[35]were closely aligned with those identified by the quantitative studies and were grouped into a single synthesised finding, and even though the other study[34] provided strategies to aid an improvement in compliance there were no findings for these.

Allocation of a person or clinician group $[35,36]$ contained two finding and was seen as a facilitator by providing a clear identity for those with responsibility for completing VTE risk assessment and prophylaxis. This was seen to facilitate by making it clear who was responsible to undertake the VTE prevention and management role, but also ensuring it was completed in a timely manner. This would also empower a person to enable them to provide reminders to responsible clinicians to facilitate compliance and ultimately improved patient outcomes.

Audit and feedback[36]was the second category and contained one finding. The reporting of both national and local statistics on morbidity and mortality associated with VTE, acted as a reminder and was used by practitioners to inform their practice.

The third category of facilitator, containing four findings was system development [36].Reminders to conducting VTE risk assessment such as development of pre-printed order and screening tools that are integrated into a systems approach or patients and family reminding clinicians, as well as organisations providing sufficient human resources to support increased mobilisation, were seen as being facilitators.

The synthesised finding generated by combining these categories was entitled 'Facilitators to improve compliance with venous thromboembolism clinical practice guidelines', with the observation that any activity that enhances and improves a clinician's compliance with evidence-based clinical practice guidelines for venous thromboembolism prevention and management can be considered as being a facilitator.

\section{Discussion}

Clinical guidelines are a tool for health professionals to make decisions in clinical practice based on research evidence[40].They are designed to support healthcare professionals in making appropriate choices in regards to patient care so as to provide optimal patient outcomes[40, 41]. Clinical practice guidelines standardise care and reduce the variations of practice within as well as between organisations, which ultimately reduces variation in patient outcomes[42]. They are developed by integrating evidence from studies in a systematic way and situating them into recommendations for practice[40].

Guideline dissemination and implementation using simple distribution of the clinical practice guidelines is insufficient to provide a change in practice[43]. Merging more than one 
strategy in the dissemination of information when implementing a new clinical practice guideline, has been suggested as the optimal approach when practice modification is required[43]. There are different forms of interventions that are used to inform healthcare professionals and patients about clinical practice guidelines and these encompass education, including the supply of education material, meetings and outreach visits, reminders and decision support methods as well as conducting audits and feedback of the results[44].

Even though venous thromboembolism clinical practice guidelines have been around for many years they do not always translate into practice[14, 15]. This leads to a variation in quality of care provided within a healthcare facility as well as between different organisations[16]. These guidelines can direct care provided and ultimately if not followed provide unfairness in expected clinical outcomes[16]. There are different reasons for clinical guidelines not being followed by healthcare professionals and knowing these reasons when developing strategies to disseminate guideline information is important as well as monitoring compliance with clinical audits[16].

All included studies identified that there was an improvement in the compliance with clinical practice guidelines after an intervention was provided. The improvement rate for the studies in level of compliance ranged from $5 \%$ to $58 \%$. The majority of interventions provided some form of education or awareness of the need to assess patients for VTE risk and to initiate prophylaxis, either by face to face sessions or reminders on the computer, patient case notes or newsletters. Therefore interventions that promoted awareness and assisted in making adhering to the guidelines easier to maintain lead to an increase in compliance with the guidelines. It was also identified that a once off intervention was not enough to sustain improved compliance over the long term, therefore multiple modes of delivery at different time intervals was seen to be more beneficial for sustained improvement. The interventions that are the most beneficial are regular education with feedback from audits, as well as clinician reminders. These three intervention supports, along with a dedicated VTE clinician, are the optimal strategies to affect practice change and sustain it for the long term.

The two qualitative studies arrived at very similar strategies for improvement with VTE prevention and management as the quantitative studies. They both discussed the need to standardize care within and across organizations. There was also an agreement that clearly identifying a clinician or practitioner group to be responsible for the prevention and management procedures would be beneficial. They identified that if a dedicated person or group were responsible for VTE procedures and protocols then care would be initiated more quickly, and reminders would not be forgotten. Providing staff with regular updates on local and national statistics that included facility compliance rates, morbidity and mortality would provide the ability for practitioners to become more informed, and therefore lead to a more active participation in VTE prevention and management. Providing evidence-based risk assessment tools and pre-printed order forms for prophylaxis, as well as regular feedback on facility audits would improve practitioner compliance. Another strategy identified was to provide more allied health staff, like physiotherapists, to support ward staff and provide qualified assistance with increasing patient mobilization.

The study that had the greatest impact on VTE compliance was conducted in Australia and the duration of the study was five years, providing evidence that long term intervention is more beneficial for sustained change and improvement[1]. This study employed a VTE nurse champion who was responsible for conducting regular education with staff including feedback of audit results. This shows that providing nurses with the knowledge and responsibility for VTE risk assessment and prophylaxis can deliver an improvement in the safety and quality of care provided to patients over time. A study in America that appointed a part-time nurse case manager for a 6 month period reported a $17 \%$ improvement in VTE guideline compliance[24]. This study would be good to follow up by appointing a full-time nurse in the VTE prevention position, and conduct it over a longer period of time. This would help to identify if the compliance percentage can be improved upon with full-time monitoring rather than part-time, and provide evidence of being able to sustain the compliance improvement.

The included studies identified that they were conducted in the metropolitan area or did not state location; therefore there were no studies that acknowledged they were undertaken in a rural or remote area. About one third of Australia's population lives outside of major cities and people in rural and remote areas face a significant health disadvantage[45]. Small rural and remote communities are identified as having higher hospitalization rates and higher incidence of health risk factors when compared to metropolitan areas[45]. Therefore it would be beneficial that studies conducted in metropolitan areas were replicated in rural and remote areas to ascertain if the same outcomes would result in these locations.

The limitations of this systematic review was that the terminology used within the studies included in this systematic review were not consistent across all studies and differed between countries as well as within countries. There were different countries, different healthcare professionals, different study designs, different health systems, guidelines made direct comparisons problematic and may limit application of conclusions to all settings. It appears that the studies were only conducted within the urban setting, and given the differences reported between urban and rural communities for other health issues it is not evident if an estimation VTE compliance is possible across all healthcare areas. Each study designed their own clinical assessment tool therefore the variability between studies may be greater as not all provided the criteria they used within their studies. The studies included in this review provided a multifaceted approach tailored to each type of healthcare professional 
which although it is optimal for that setting and professional group it may not be replicated with other healthcare professionals. The search strategy was limited by studies only being in the English language which may potentially have resulted in the failure to identify all relevant studies.

\section{Conclusion}

Although VTE risk assessment and prophylaxis is supported by several evidence based guidelines, clinician use of these guidelines is inconsistent. Identified reasons for this include: lack of awareness of clinical practice guidelines, confusion as to which clinical group are the target of the guidelines. Costs, in terms of financial, time and resources also acted as barriers.

Potentially many of the identified barriers could be overcome with strategies that include regular and updated education (particularly that which included local as well as national statistics on VTE morbidity and mortality), having a dedicated VTE person (a VTE Champion) with the responsibility of VTE assessment/reporting/education across a facility or healthcare service.

\section{Acknowledgements}

This systematic review formed partial submission for the degree award of Masters of Clinical Science through the Joanna Briggs Institute, The University of Adelaide and was not supported by any specific grant.

The authors would like to thank

Associate Supervisor: Dr Gary Misan BPharm PhD, Associate Research Professor, Centre for Rural Health and Community Development University of South Australia, SA

Associate Supervisor: Dr David Tivey PhD, Research Fellow, The Joanna Briggs Institute, Faculty of Health Sciences, The University of Adelaide, SA

Catherine Nairn, Librarian, University of South Australia, Centre for Regional Engagement

University of South Australia, Centre for Regional Engagement for supporting this research.

\section{Disclosure of Conflict of Interest}

No conflict of interests.

\section{REFERENCES}

[1] R. Collins, L. MacLellan, H. Gibbs, D. MacLellan, J. Fletcher. Venous Thromboembolism prophylaxis: The role of the nurse in changing practice and saving lives, Australian Journal of Advanced Nursing. Vol. 27, No. 3, 83-9, 2010.

[2] National Health and Medical Research Council (NHMRC). Clinical Practice Guideline for the prevention or venous
Thromboembolism (deep vein thrombosis and pulmonary embolism) in patients admitted to Australian Hospitals,NHMRC, Melbourne, 2009.

[3] Access Economics. The burden of venous thromboembolism in Australia, Report for the Australian and New Zealand Working Party on the management and prevention of venous thromboembolism, Access Economics Pty Limited, 2008.

[4] National Health and Medical Research Council. Stop the Clot: Integrating VTE prevention guideline recommendations into routine hospital care, NHMRC, 2011.

[5] D. MacDougall, A. Feliu, S. Boccuzzi, J. Lin. Economic Burden of Deep-vein thrombosis, pulmonary embolism, and post-thrombotic syndrome, American Journal of Health-system Pharmacy, Vol. 63, No. 20, S5-S15.2006

[6] R. Coombes. Venous thromboembolism caused 25000 deaths in a year, says MP's, British Medical Journal, Vol. 330, 559, 2005.

[7] S. Moll, N. Mackman. Venous Thromboembolism: A need for more Public Awareness and research into mechanisms, Arteriosclerosis, Thrombosis and Vascular Biology, American Heart Association, Vol. 28, 367-369, 2008.

[8] J. Hirsch, J. Hoak. Management of Deep Vein Thrombosis and Pulmonary Embolism, Circulation, Vol. 93, No. 12, 2212-45, 1996.

[9] A. Cohen, V. Tapson, J. Bergmann, S. Goldhaber, A. Kakkar, B. Deslandes, W. Huang, M. Zayaruzny, L. Emery, F. Anderson Jr. Venous thromboembolism risk and prophylaxis in the acute hospital care setting (ENDORSE study): a multinational cross-sectional study, The Lancet, Vol. 371, No. 9610, 387-394, 2008.

[10] N. Zakai, P. Callas, A. Repp, M. Cushman. Venous thrombosis risk assessment in medical inpatients: the medical inpatients and thrombosis (MITH) study, Journal of Thrombosis and Haemostasis, Vol. 11, 634-641, 2013.

[11] N. Hairon. New risk assessment tool aims to help nurses prevent VTE, NursingTimesnet, 2008.

[12] E. Nutescu. Assessing, preventing, and treating venous thromboembolism: Evidence-based approaches, American Journal of Health-system Pharmacy, Vol. 64, No. 11, Suppl 7, 2007.

[13] C. Farquhar, E. Kofa, J. Slutsky. Clinicians' attitudes to clinical practice guidelines: a systematic review, The Medical Journal of Australia, Vol. 177, No. 9, 502-506, 2002.

[14] J. Ploeg, B. Davies, N. Edwards, W. Gifford, P. Miller. Factors influencing Best-practice guideline implementation: Lessons learned from administrators, nursing staff, and project, leaders, Worldviews on Evidence-Based Nursing, fourth quarter, 210-219, 2007.

[15] R. Grol, H. Buchan. Clinical guidelines: what can we do to increase their use? MJA, Vol. 185, No. 6, 301-302, 2006.

[16] W. McGuire, P. Fowlie. Bridging the gaps: getting evidence into practice, CMJA, Vol. 181, No. 8, 457-458, 2009.

[17] R. Tooher, P. Middleton, C. Pham, R. Fitridge, S. Rowe, W. Babidge, and G. Maddern. A systematic review of Strategies to improve prophylaxis for venous thromboembolism in hospitals, Annals of Surgery, Vol. 241, No. 3, 397-415, 2005.

[18] J. Duff, K. Walker, A. Omari. Translating venous thromboembolism (VTE) prevention evidence into practice: a multidisciplinary evidence implementation project. 
Worldviews Evidence Based Nursing, Vol. 8, No. 1, 30-39, 2011.

[19] L. Lees, M. McAuliffe. Venous thromboembolism risk assessments in acute care, Nursing Standard, Vol. 24, No. 22 , $35-41,2010$.

[20] B. Sharif-Kashani, S. Raeissi, B. Bikdeli, P. Shahabi, N Behzadnia, L. Saliminejad, M. Samiei-Nejad, F. Nasiri, M. Khayyami, B. Forootan, S. Pozhan, M-R. Masjedi. Sticker reminders improve thromboprophylaxis appropriateness in hospitalized patients, Thrombosis Research, Vol. 126, No. 3, 211-216, 2010.

[21] F. Gaylis, S. Van, M. Daneshvar, G. Gaylis, J. Gaylis, R. Sheela, E. Stern, P. Hanson and R. Sur. Preprinted standardized orders promote venous thromboembolism prophylaxis compared with traditional handwritten orders: an endorsement of standardized evidence-based practice, American Journal of Medical Quality, Vol. 25, No. 6, 449-456, 2010.

[22] D. Sobieraj. Development and implementation of a program to assess medical patients' need for venous thromboembolism prophylaxis, American Journal of Health-System Pharmacy,Vol. 65, No. 18, 1755-1760, 2008.

[23] R. Bullock-Palmer, S. Weiss, C. Hyman. Innovative approaches to increase deep vein thrombosis prophylaxis rate resulting in a decrease in hospital-acquired deep vein thrombosis at a tertiary-care teaching hospital, Journal of Hospital Medicine,Vol. 3, No. 2, 148-55, 2008.

[24] T. Schiro, J. Sakowski, R. Romanelli, T. Jukes, J. Newman, A. Hudnut, and $\mathrm{T}$. Leonard. Improving adherence to best-practice guidelines for venous thromboembolism risk assessment and prevention, American Journal of Health-System Pharmacy,Vol. 68, No. 22, 2184-2189, 2011.

[25] F. Li, K. Walker, E. McInnes, J. Duff. Testing the effect of a targeted intervention on nurses' compliance with "best practice" mechanical venous thromboembolism prevention, Journal of Vascular Nursing, Vol. 28, No. 3, 92-96, 2010.

[26] J. Al-Tawfiq, B. Saadeh. Improving adherence to venous thromoembolism prophylaxis using multiple interventions, Annals of Thoracic Medicine,Vol. 6, No. 2, 82-84, 2011.

[27] G. Shedd, C. Franklin, A. Schumacher, D. Green. Improving inpatient venous thromboembolism prophylaxis, Southern Medical Journal,Vol. 101, No. 12, 1209-1215, 2008

[28] E. Janus, A. Bassi, D. Jackson, H. Nandurka, M. Yates. Thromboprophylaxis use in medical and surgical inpatients and the impact of an electronic risk assessment tool as part of a multi-factorial intervention, A report on behalf of the elVis study investigators, Journal of Thrombosis and Thrombolysis, Vol. 32, No. 3, 279-287, 2011.

[29] S. Novis, G. Havelka, D. Ostrowski, B. Levin, L. Blum-Eisa, J. Prystowsky, and M. Kibbe. Prevention of thromboembolic events in surgical patients through the creation and implementation of a computerized risk assessment program, Journal of Vascular Surgery,Vol. 51, No. 3, 648-654, 2010.

[30] M. Moote, M. Englesbe, V. Bahl, H. Hu, M. Thompson, J. Kubus, D. Campbell Jr. PA-driven VTE risk assessment improves compliance with recommended prophylaxis, Journal of the American Academy of Physician Assistants (JAAPA),Vol. 23, No. 6, 27-35, 2010.

[31] P. Dobesh, Z. Stacy. Effect of a clinical pharmacy education program on improvement in the quantity and quality of venous thromboembolism prophylaxis for medically ill patients, Journal of managed care pharmacy,Vol. 11, No. 9, 755-762, 2005.

[32] B. Kent, P. Nadarajan, N. Akasheh, I. Sulaiman, S. Karim, S. Cooney, S. Lane, E. Moloney. Improving venous thromboembolic disease prophylaxis in medical inpatients: A role for education and audit, Irish Journal of Medical Science,Vol. 180, No. 1, 163-166, 2011.

[33] S. Baroletti, K. Munz, J. Sonis, J. Fanikos, K. Fiumara, M. Paterno, S. Goldhaber. Electronic alerts for hospitalized high-VTE risk patients not receiving prophylaxis: a cohort study, Journal of Thrombosis and Thrombolysis, Vol. 25, 146-150, 2008

[34] G. Maynard, T. Morris, I. Jenkins, S. Stone, J. Lee, M. Renvall, E. Fink, R. Schoenhaus. Optimizing Prevention of Hospital-acquired Venous Thromboembolism (VTE): Prospective Validation of a VTE Risk Assessment Model, Journal of Hospital Medicine,Vol. 5, No. 1, 10-18, 2010.

[35] N. Chapman, S. Lazar, M. Fry, M. Lassere, B. Chong. Clinicians adopting evidence based guidelines: a case study with thromboporphylaxis, BMC Health Services Research,Vol. 11, 240, 2011.

[36] D. Cook, A. Tkaczyk, K. Lutz, J. McMullin, R. Haynes, J. Douketis. Thromboprophylaxis for hospitalized medical patients: a multicentre qualitative study, Journal of Hospital Medicine,Vol. 4, No. 5, 269-275, 2009.

[37] Department of Health, Chief Medical Officer. Report of the Independent Expert Working Group on the Prevention of Venous Thromboembolism in Hospitalised patients,Department of Health, London, 2007.

[38] American College of Chest Physicians. The American College of Chest Physicians Guidelines on antithrombotic and thrombolytic therapy: ACCP Evidence-based Clinical Practice Guidelines, Chest,Vol. 133, No. 6 suppl b-c, 2008.

[39] The Australia \& New Zealand Working Party on the Management and Prevention of Venous Thromboembolism. Prevention of Venous Thromboembolism, Innovations HEM, Australia, 2010.

[40] S. Osborne, J. Webster. Development and use of clinical guidelines, In: M. Courtney, H. McCutcheon, eds, Using Evidence to guide nursing practice, Churchill Livingstone Elsevier, Sydney, 59-73, 2010.

[41] M. Eccles, J. Grimshaw. Selecting, presenting and delivering clinical guidelines: are there any "magic bullets"? Medical Journal of Australia.Vol. 180, No. 6, S52-S54, 2004.

[42] T. Nakayama. What are "clinical practice guidelines"? Journal of Neurology,Vol. 254, Suppl 5, 2-7, 2007.

[43] B. Fervers, J. Carretier, A. Bataillard. Clinical practice guidelines, Journal of Visceral Surgery,Vol. 147, e341-e349, 2010 .

[44] G. Barosi. Strategies for dissemination and implementation of guidelines, Neurological Sciences,Vol. 27, No. 3, S231-S234, 2006.

[45] J. Wakerman, J. Humphreys, R. Wells, P. Kuipers, P. Entwistle, J. Jones. Primary health care delivery models in rural and remote Australia - a systematic review, BMC Health Services Research,Vol. 8, No. 1, 2008. 